\title{
Stabilization of Planar Collective Motion With Limited Communication
}

\author{
Rodolphe Sepulchre, Member, IEEE, Derek A. Paley, Member, IEEE, and Naomi Ehrich Leonard, Fellow, IEEE
}

\begin{abstract}
This paper proposes a design methodology to stabilize relative equilibria in a model of identical, steered particles moving in the plane at unit speed. Relative equilibria either correspond to parallel motion of all particles with fixed relative spacing or to circular motion of all particles around the same circle. Particles exchange relative information according to a communication graph that can be undirected or directed and time-invariant or timevarying. The emphasis of this paper is to show how previous results assuming all-to-all communication can be extended to a general communication framework.
\end{abstract}

Index Terms-Cooperative control, geometric control, multiagent systems, stabilization.

\section{INTRODUCTION}

$\mathbf{T}$ HE DEVELOPMENT of versatile and scalable methodology to control collective motion for multiagent systems is driven by a growing number of engineering applications that depend on the coordination of a group of individually controlled systems. The various applications, which impose diverse operational constraints and performance requirements, include formation control of unmanned aerial vehicles (UAVs) [1], [2] and spacecraft [3], cooperative robotics [4] [5], and sensor networks [6], [7]. In this paper, we are strongly motivated by the application of autonomous underwater vehicles (AUVs) as mobile sensors to collect oceanographic measurements in formations or patterns that yield maximally information-rich data sets; see, e.g., [8] and [9]. Such patterns are designed to match the measurement density in space and time to the characteristic scales of the oceanographic process of interest. Coordinated, periodic trajectories such as the ones studied in this paper provide a means to collect measurements with the desired spatial and temporal separation.

Motivated by these issues, we consider, in the present paper, the design of stable collective patterns in a model of identical planar particles introduced in [10]. The particles move at constant speed and are subject to steering controls that change their direction of motion. In addition to a phase variable that models the orientation of the velocity vector, the state of each particle includes its position in the plane. The synchrony of the collec-

Manuscript received August 31, 2006; revised March 27, 2007. Recommended by Associate Editor F. Bullo.

R. Sepulchre is with the Department Electrical Engineering and Computer Science, Institut Montefiore B28, Universite de Liège, B-4000 Liège, Belgium. (e-mail: r.sepulchre@ulg.ac.be).

D. A. Paley is with the Department of Aerospace Engineering, University of Maryland, College Park, MD 20742 USA (e-mail: dpaley@umd.edu).

N. E. Leonard is with the Department of Mechanical and Aerospace Engineering, Princeton University, Princeton, NJ 08544 USA (e-mail: naomi@princeton.edu).

Digital Object Identifier 10.1109/TAC.2008.919857 tive motion is thus measured both by the relative phasing and the relative spacing of particles.

In recent work [11], we proposed a versatile design methodology that specifies collectives as minima of artificial potentials. These potentials are used as Lyapunov functions for the design of gradient-like control laws that make them decreasing along the solutions of the closed-loop system. The resulting control laws exhibit a number of desirable properties, but they require all-to-all communication, that is, the control law of a given particle requires relative information from all other particles. All-to-all communication is an assumption that is unrealistic in a number of applications. In large sensor networks, each agent is expected to exchange information with a limited number of neighbors only. The interplay between the information flow among particles and the individual agent dynamics then becomes a central issue of the collective design. Even if all-to-all communication is available, limited use of communication may be advantageous for performance. The objective of the present paper is to address this issue in the framework of the planar particle model [10] and to show that the results previously obtained in the framework of all-to-all communication [11] can be recovered in a systematic way under quite general assumptions on the network communication.

Two different approaches are considered to address the limited communication topology. A first proposed approach is to generalize the design of artificial potentials in such a way that the resulting control laws respect the communication constraints. Encoding the communication constraints in a graph, we propose the design of potentials based on the graph Laplacian, and show that these potentials reduce to the previously proposed all-to-all potentials in the particular case of a complete graph. This approach requires the communication topology to be time-invariant and connected. The gradient-type control laws proposed in the present paper further require the communication to be bidirectional. This generalization is relevant, for instance, in design settings, where the topology of the communication is a design parameter, but where the number of communication links is limited by technological constraints. The framework has been previously considered in [12]-[14], for instance, where the authors focused on circulant graphs and studied the stability of various circular formations also considered in the present paper.

A second approach is to generalize the design of collectives in a framework where the communication is possibly time-varying, unidirectional, and not fully connected at any given instant of time. The possibility to achieve synchronization under such general assumptions on the communication has been extensively addressed in the recent literature on consensus algorithms [15]-[19]. In particular, the analysis tools proposed 
in [16] and [20] are appropriate to study convergence issues in the present framework under weak assumptions on the communication graph. The application of these tools imposes restrictions on the system dynamics, and, more fundamentally, on the Euclidean nature of the underlying state space. As a consequence, only local results have been available for non-Euclidean state spaces such as those encountered in phase-oscillators models [16], [21] or in the present particle model [22]. However, a recent result [23], [24] shows how consensus estimators designed for Euclidean space [25] can be used in the closed-loop system dynamics to obtain globally convergent consensus algorithms in phase models. We use this idea to generalize our previously proposed all-to-all design [11] to general communication topologies. In this approach, the weakened assumptions on the communication topology are compensated for by an increased exchange of information between communicating particles. Communicating particles indeed not only exchange their relative configuration variables, but also their relative estimates of averaged quantities used in centralized designs.

The paper follows the logical structure of our companion paper [11]. Each section briefly summarizes the corresponding all-to-all result of [11], then provides the generalization to: 1) time-invariant and undirected communication and 2) uniformly connected communication, which allows for time-varying and directed communication. Section II reviews the geometric properties of the considered particle model. Section III focuses on a simpler phase model that controls only the relative orientation of the particle velocities. In Section IV, we consider the stabilization of parallel and circular formations, which are two types of relative equilibria of the full particle model. The next two sections elaborate on these basic results by proposing symmetrybreaking control laws that either reduce the dimension of the equilibrium set in shape space and isolate symmetric patterns (Section V) or achieve stabilization in the absolute space by connecting the network to a reference particle (Section VI). Section VII illustrates an application of the results in the framework of the ocean sampling project that motivated many of the developments proposed in the present paper. Section VIII illustrates an application of the results in the framework of a proximity communication graph that involves unidirectional and time-varying communication links. Conclusions are presented in Section IX.

\section{Model And Problem Statement}

\section{A. Model of Steered Particles in the Plane}

We consider a continuous-time model of $N$ identical particles (of unit mass) moving in the plane at unit speed and subject to steering control [1]

$$
\begin{aligned}
& \dot{r}_{k}=e^{i \theta_{k}} \\
& \dot{\theta}_{k}=u_{k}, \quad k=1, \ldots, N .
\end{aligned}
$$

In complex notation, the vector $r_{k}=x_{k}+i y_{k} \in \mathbb{C} \approx \mathbb{R}^{2}$ denotes the position of particle $k$, and the angle $\theta_{k} \in S^{1}$ denotes the orientation of its (unit) velocity vector $e^{i \theta_{k}}=$ $\cos \theta_{k}+i \sin \theta_{k}$. We call $\theta_{k}$ the phase of particle $k$. The scalar $u_{k}$ is the steering control for particle $k$. The model (1) reflects second-order dynamics of particles with forcing only in the direction normal to velocity (steering control), i.e., $\ddot{r}_{k}=\dot{\theta}_{k}\left(i \dot{r}_{k}\right)$ with $\dot{r}_{k}$ of unit length. We use a bold variable without index to denote the corresponding $N$-vector, e.g., $\boldsymbol{\theta}=\left(\theta_{1}, \ldots, \theta_{N}\right)^{T}$ and $\boldsymbol{u}=\left(u_{1}, \ldots, u_{N}\right)^{T}$. In the absence of steering control $\left(\dot{\theta}_{k}=0\right)$, each particle moves at unit speed in a fixed direction and its motion is decoupled from the other particles. We study the design of various feedback control laws that result in coupled dynamics and closed-loop convergence to different types of organized or collective motion.

The model (1) has been recently studied by Justh and Krishnaprasad [1]. These authors have emphasized the Lie group structure that underlies the state space. The configuration space consists of $N$ copies of the group $S E(2)$. When the control law only depends on relative phases and relative positions, the closed-loop vector field is invariant under an action of the symmetry group $S E(2)$ and the closed-loop dynamics evolve on a reduced quotient manifold. This $(3 N-3)$-dimensional manifold is called the shape space, and it corresponds to the space of all relative phases and relative positions. For all pairs $j$ and $k$, we use shape variables $\theta_{k}-\theta_{j}$ and $\left(r_{k}-r_{j}\right) e^{-i \theta_{k}}$, which are the phase and position of particle $j$ relative to the phase and position of particle $k$, respectively. Equilibria of the reduced dynamics are called relative equilibria and include parallel motions, characterized by a common orientation for all the particles (with arbitrary relative spacing), and circular motions, characterized by circular orbits of the particles around a fixed point [1].

\section{B. Graph Representation of the Communication Limitations}

It is common in a multiagent framework to describe the communication between particles by means of a graph. Each of the $N$ nodes in the graph represents a particle in the model. A (weighted) edge from $k$ to $j$ indicates a communication link from particle $k$ to particle $j$. The resulting weighted digraph (directed graph) is denoted by $G=(\mathcal{I}, \mathcal{E}, A)$, where $\mathcal{I}=\{1, \ldots, N\}$ is the set of node indices, $\mathcal{E} \subseteq \mathcal{I} \times \mathcal{I}$ is the set of edges, and $A$ is a weighted adjacency matrix with nonnegative elements $a_{j k}$. The element $a_{j k}$ is bounded away from zero when there is a communication link (i.e., information passing) from $k$ to $j$, and $a_{j k}=0$ when there is no communication link from $k$ to $j$. We assume that there are no self-cycles, i.e., $a_{k k}=0, \forall k \in \mathcal{I}$.

The digraph $G$ is called strongly connected if and only if any two distinct nodes of the graph can be connected via a path that follows the direction of the edges of the digraph.

The in-degree and out-degree of node $k$ are defined as $d_{k}^{\text {in }}=$ $\sum_{j=1}^{N} a_{k j}$ and $d_{k}^{\text {out }}=\sum_{j=1}^{N} a_{j k}$, respectively. The digraph $G$ is said to be balanced if the in-degree and the out-degree of each node are equal, that is

$$
\sum_{j} a_{k j}=\sum_{j} a_{j k} \quad \forall k \in \mathcal{I} .
$$


The graph Laplacian $L$ associated to the graph $G$ is a square matrix defined as

$$
l_{k j}= \begin{cases}\sum_{i} a_{k i}, & j=k \\ -a_{k j}, & j \neq k .\end{cases}
$$

The $k$ th row of $L$ is denoted by $L_{k}$. By construction, the Laplacian matrix has zero row sums, that is, $L \mathbf{1}=0$, where $\mathbf{1}=(1, \ldots, 1)^{T}$ is an $N \times 1$ matrix. Several properties of the graph translate into matrix properties of its Laplacian $L$. In particular, the following properties are relevant in the framework of the present paper:

1) the graph $G$ is undirected if and only if $L$ is symmetric;

2) if the graph $G$ is strongly connected, then 1 spans the kernel of $L$, i.e., $L \boldsymbol{x}=0$ if and only if $\boldsymbol{x}=\mathbf{1} x_{0}$ [18, Th. 5]; and

3) the graph $G$ is balanced if and only if $L+L^{T} \geq 0$ [20, Proposition 1].

For further background on spectral graph theory, see, for example, [26].

A particular generalization of the all-to-all communication topology that we consider in the present paper is the fixed and connected topology corresponding to a $d_{0}$-circulant graph in which each node is connected to $d_{0}$ other nodes, where $d_{0}$ is a fixed integer in the interval $[2, N-1]$. Both the adjacency and Laplacian matrices of a circulant graph are circulant, i.e., they are completely defined by their first row. Each subsequent row of a circulant matrix is the previous row shifted one position to the right with the first entry equal to the last entry of the previous row. For example, the all-to-all topology is $(N-1)$-circulant and the ring topology is 2-circulant. The following lemma (see, for instance, [27]) will be useful in various proofs.

Lemma 1: Let $L$ be the Laplacian of a $d_{0}$-circulant graph with $N$ vertices. Set $\phi_{k}=(k-1) 2 \pi / N$ for $k=1, \ldots, N$. Then, the vectors

$$
\boldsymbol{f}^{(l)}=e^{i(l-1) \phi}, \quad l=1, \ldots, N
$$

define a basis of $N$ orthogonal eigenvectors of $L$. The unitary matrix $F$ whose columns are the $N$ (normalized) eigenvectors $(1 / \sqrt{N}) \boldsymbol{f}^{(l)}$ diagonalizes $L$, that is, $L=F \Lambda F^{*}$, where $\Lambda=$ $\operatorname{diag}\left\{0, \lambda_{2}, \ldots, \lambda_{N}\right\} \geq 0$ is the (real) diagonal matrix of the eigenvalues of $L$.

It is of both theoretical and practical interest to consider time-varying communication topologies; in a network of moving particles, existing links can fail and new links can appear as other particles leave or enter an effective range of detection. Time-varying communication topologies will be described by a time-varying $\delta$-digraph $G(t)=(\mathcal{I}, \mathcal{E}(t), A(t))$, where the elements of $A(t)$ are bounded and satisfy some threshold $\delta>0$, that is, $a_{k j}(t)=$ 0 in the absence of a communication link and $a_{k j}(t) \geq \delta$ in the presence of a communication link. The set of neighbors of node $k$ at time $t$ is denoted by $\mathcal{N}_{k}(t):=\left\{j \in \mathcal{I}: a_{k j}(t) \geq \delta\right\}$.

Consider a graph $G(t)=(\mathcal{I}, \mathcal{E}(t), A(t))$. A node $k$ is said to be connected to node $j(j \neq k)$ in the interval $I=\left[t_{a}, t_{b}\right]$ if there is a path from $k$ to $j$, which respects the orientation of the edges for the directed graph $\left(\mathcal{I}, \cup_{t \in I} \mathcal{E}(t), \int_{I} A(\tau) d \tau\right)$. G(t) is said to be uniformly connected [20] if there exists an index $k$ and a time horizon $T>0$ such that, for all $t$, node $k$ is connected to all the other nodes across $[t, t+T]$.

\section{Phase Synchronization And Phase Balancing}

\section{A. All-to-All Communication}

All control laws in this paper are based on gradientlike stabilization of collectives specified as critical points of well-chosen potentials. The inner product $\langle\cdot, \cdot\rangle$ is defined by $\left\langle z_{1}, z_{2}\right\rangle=\operatorname{Re}\left\{z_{1}^{*} z_{2}\right\}$ for $z_{1}, z_{2} \in \mathbb{C}$, where ${ }^{*}$ denotes the complex conjugate. For vectors, we use the analogous boldface notation $\langle\boldsymbol{w}, \boldsymbol{z}\rangle=\operatorname{Re}\left\{\boldsymbol{w}^{*} \boldsymbol{z}\right\}$ for $\boldsymbol{w}, \boldsymbol{z} \in \mathbb{C}^{N}$, where ${ }^{*}$ here denotes the conjugate transpose. We first recall a basic result from our previous paper [11], which characterizes the critical points of the potential $U_{m}(\boldsymbol{\theta})$ defined as

$$
U_{m}=\frac{N}{2}\left|p_{m \theta}\right|^{2}
$$

where $p_{m \theta}$ is the $m$ th moment of the phase distribution on the unit circle defined as

$$
p_{m \theta}=\frac{1}{m N} \sum_{k=1}^{N} e^{i m \theta_{k}}, \quad m=1,2, \ldots
$$

Theorem 1 (synchronization and balancing): Let $1 \leq m \in \mathbb{N}$. The potential $U_{m}=(N / 2)\left|p_{m \theta}\right|^{2}$ reaches its unique minimum when $p_{m \theta}=0$ (balancing modulo $2 \pi / m$ ) and its unique maximum when the phase difference between any two phases is an integer multiple of $2 \pi / m$ (synchronization modulo $2 \pi / m$ ). All other critical points of $U_{m}$ are isolated in the shape manifold $T^{N} / S^{1}$ and are saddle points of $U_{m}$.

Proof: See [11, Th. 5].

The significance of Theorem 1 for the collective model (1) is as follows: for $m=1$, the potential $U_{1}(\boldsymbol{\theta})$ reaches its maximum when all phases synchronize. This state is characteristic of parallel formations in the collective model (1), i.e., all particles move in the same direction. In contrast, the potential $U_{1}(\boldsymbol{\theta})$ reaches its minimum when all phases balance. This state is characteristic of a fixed center of mass in the collective model because $p_{\theta}:=p_{1 \theta}=(1 / N) \sum_{k=1}^{N} \dot{r}_{k}$. The parameter $\left|p_{\theta}\right|$ is a classical measure of synchrony of the phase variables $\boldsymbol{\theta}$ in the literature on coupled oscillators. In the context of the Kuramoto model, $p_{\theta}$ has been called the complex order parameter [28].

For $m>1$, the interpretation of the critical points of $U_{m}$ is similar modulo $2 \pi / m$, that is, when phases differing by $2 \pi / m$ are identified. The use of potentials $U_{m}$ for $m>1$ is postponed until Section V, where we address the stabilization of particular symmetric phase arrangements. Fig. 1 provides a simple illustration of the role of balancing and synchronizing different moments to attain specific phase arrangements.

To achieve stabilization of minima or maxima of the potential $U_{m}$, it is natural to consider the gradient control $\boldsymbol{u}=$ $-K \operatorname{grad} U_{m}$, i.e.

$$
u_{k}=-K\left\langle p_{m \theta}, i e^{i m \theta_{k}}\right\rangle=\frac{K}{m N} \sum_{j=1}^{N} \sin \left(m\left(\theta_{k}-\theta_{j}\right)\right)
$$




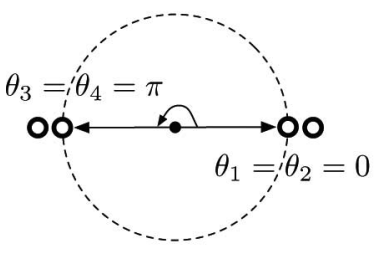

(a)

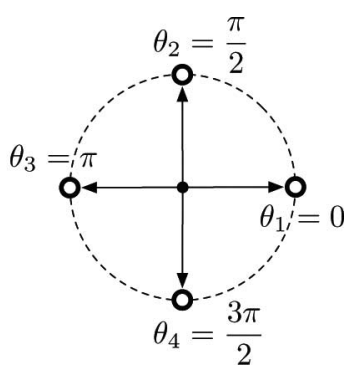

(b)
Fig. 1. Phase synchronization and balancing examples for $N=4$. (a) Phase arrangement is balanced modulo $2 \pi$ and synchronized modulo $\pi$; i.e., $p_{\theta}=$ 0 and $2\left|p_{2 \theta}\right|=1$. (b) Phase arrangement is balanced modulo $2 \pi$, balanced modulo $\pi$, and synchronized modulo $\pi / 2$; i.e., $p_{\theta}=p_{2 \theta}=0$ and $4\left|p_{4 \theta}\right|=1$.

where $K \neq 0$. The resulting closed-loop dynamics corresponds to either a descent or ascent algorithm depending on the sign of the gain $K$, leading to the following result as a direct consequence of Theorem 1 . As a convention throughout the paper, we associate negative gains $(K<0)$ to synchronization controls and positive gains $(K>0)$ to balancing controls.

Corollary 1: The phase model $\dot{\boldsymbol{\theta}}=\boldsymbol{u}$ with the gradient control (5) forces convergence of all solutions to the critical set of $U_{m}$. If $K<0$, then only the set of synchronized states is asymptotically stable, and every other equilibrium is unstable. If $K>0$, then only the balanced set $p_{m \theta}=0$ is asymptotically stable and every other equilibrium is unstable.

The all-to-all sinusoidal coupling (5) with $m=1$ is a frequently studied coupling in the literature of coupled oscillators [28]-[30]. However, it requires all-to-all communication, an assumption that we relax in the following sections.

\section{B. Time-Invariant and Undirected Communication Graphs}

Let $P=I_{N}-(1 / N) \mathbf{1 1}{ }^{T}$. Then, $P e^{i m \boldsymbol{\theta}}=e^{i m \boldsymbol{\theta}}-m p_{m \theta} \mathbf{1}$. One deduces the equality

$$
\left\|P e^{i m \boldsymbol{\theta}}\right\|^{2}=\left\langle e^{i m \boldsymbol{\theta}}, P e^{i m \boldsymbol{\theta}}\right\rangle=N\left(1-m^{2}\left|p_{m \theta}\right|^{2}\right)
$$

which implies that the quadratic form $\left\langle e^{i m \boldsymbol{\theta}}, P e^{i m \boldsymbol{\theta}}\right\rangle$ has the same critical points as the phase potential $U_{m}(\boldsymbol{\theta})$. Because $P$ is $(1 / N$ times) the Laplacian matrix of the complete graph, the identity (6) suggests to replace the optimization of $U_{m}(\theta)$ in the previous section by the optimization of

$$
W_{m}(\boldsymbol{\theta})=\frac{1}{m^{2}} Q_{L}\left(e^{i m \boldsymbol{\theta}}\right):=\frac{1}{2 m^{2} d_{\max }}\left\langle e^{i m \boldsymbol{\theta}}, L e^{i m \boldsymbol{\theta}}\right\rangle
$$

where we denote by $Q_{L}(s)$ the quadratic form in $s \in \mathbb{C}^{N}$ and $d_{\max }=\max _{k \in \mathcal{I}}\left\{d_{k}^{\text {in }}\right\}$. Notice that $W_{m}(\boldsymbol{\theta})=(N /(N-$ 1)) $\left.\left(N /\left(2 m^{2}\right)\right)-U_{m}(\boldsymbol{\theta})\right)$ in the case of a complete graph in which case, both $W_{m}(\boldsymbol{\theta})$ and $U_{m}(\boldsymbol{\theta})$ have the same critical points.

Suppose that $L$ is the Laplacian of an undirected graph with unit edge weights, which implies $L=L^{T}$ and $a_{k j} \in\{0,1\}$. Then, the significance of optimizing the potential $W_{m}$ instead of $U_{m}$ is that the gradient control

$$
u_{k}=K \frac{\partial W_{m}}{\partial \theta_{k}}=\frac{K}{m d_{\max }} \sum_{j \sim k} \sin \left(m\left(\theta_{k}-\theta_{j}\right)\right)
$$

is adapted to the topology of the communication graph. That is, the control $u_{k}$ only uses the relative information from the communicating neighbors $j \in \mathcal{N}_{k}$, denoted by $j \sim k$.

The synchronized state modulo $2 \pi / m$ is a global minimum of $W_{m}$ provided that $G$ is balanced. However, $W_{m}$ may possess other (local) minima, and the (local) maxima do not necessarily correspond to balanced states. A meaningful generalization of Theorem 1 holds for $d_{0}$-circulant graphs.

Theorem 2: If $L$ is the Laplacian of a connected and balanced graph, then the global minimum of $W_{m}(\boldsymbol{\theta})$ is synchronized modulo $2 \pi / m$, i.e., $e^{i m \boldsymbol{\theta}}=e^{i \theta_{0}} \mathbf{1}$, where $\theta_{0} \in S^{1}$. If the graph is $d_{0}$-circulant, then the global maximum of $W_{m}(\boldsymbol{\theta})$ is balanced modulo $2 \pi / m$, i.e., $\mathbf{1}^{T} e^{i m \boldsymbol{\theta}}=0$.

Proof: If $L$ is the Laplacian of a balanced graph, then $\langle\boldsymbol{z}, L \boldsymbol{z}\rangle \geq 0$ for all $\boldsymbol{z} \in \mathbb{C}^{N}$. If the graph is also connected, then $\langle\boldsymbol{z}, L \boldsymbol{z}\rangle=0$ if and only if $\boldsymbol{z}=\mathbf{1} z_{0}$, which proves that the global minimum of $W_{m}$ is reached (only) for $e^{i m \boldsymbol{\theta}}=e^{i \theta_{0}} \mathbf{1}$. If the graph is $d_{0}$-circulant, then Lemma 1 provides the following equivalent expression for the phase potential (7):

$$
W_{m}(\boldsymbol{\theta})=\frac{1}{2 m^{2} d_{0}}\langle\boldsymbol{v}, \Lambda \boldsymbol{v}\rangle=\frac{1}{2 m^{2} d_{0}} \sum_{k=2}^{N}\left|v_{k}\right|^{2} \lambda_{k}
$$

where $\boldsymbol{v}=F^{*} e^{i m \boldsymbol{\theta}}$. Because $\|\boldsymbol{v}\|=\left\|e^{i m \boldsymbol{\theta}}\right\|=\sqrt{N}$, an upper bound is provided by

$$
W_{m}(\boldsymbol{\theta})=\frac{1}{2 m^{2} d_{0}}\langle\boldsymbol{v}, \Lambda \boldsymbol{v}\rangle \leq \frac{N}{2 m^{2} d_{0}} \lambda_{\max } .
$$

This bound is attained by selecting $e^{i m \boldsymbol{\theta}}$ as the eigenvector of $L$ associated with the maximum eigenvalue. This vector is orthogonal to the mimimal eigenvector $\mathbf{1}$, that is, it satisfies $\mathbf{1}^{T}$ $e^{i m \boldsymbol{\theta}}=0$.

The assumptions of Theorem 2 do not require bidirectionality of the communication graph. This additional property is, nevertheless, necessary to implement the gradient control law (8).

\section{Uniformly Connected Communication Graphs}

The goal of this section is to extend the stabilization of synchronized or balanced states under weaker assumptions on the communication graph. We first recall a general result that holds in the Euclidean space $\mathbb{C}^{N}$.

Proposition 1: Let $G(t)$ be a uniformly connected $\delta$-digraph and $L(t)$ the corresponding Laplacian matrix. In the linear timevarying system

$$
\dot{\boldsymbol{x}}=-L(t) \boldsymbol{x}, \boldsymbol{x} \in \mathbb{C}^{N}
$$

the set of synchronized states is uniformly exponentially stable. In particular, each solution of (11) exponentially converges to a synchronized state $\bar{x} \mathbf{1}$. If $G(t)$ is balanced, then the asymptotic consensus $\bar{x} \in \mathbb{C}$ is the average of the initial conditions $(1 / N) \sum_{k=1}^{N} x_{k}(0)$.

Proposition 1 shows that synchronization is achieved under weak assumptions on the communication graph when the state evolves in an Euclidean space. There is a vast recent literature on the convergence properties of consensus algorithms of the type (11). We refer the reader to the recent paper [16] for a proof 
of Proposition 1 (the setup in [20] is even more general since it considers communication delays).

When the graph $G$ is fixed and undirected, (11) is a gradient system for the quadratic form $(1 / 2)\langle\boldsymbol{x}, L \boldsymbol{x}\rangle$. It is tempting to consider the analog of (11) on the torus. The gradient expression (8) suggests the generalization

$$
\dot{\theta}_{k}=\frac{K}{d_{\max }}\left\langle L_{k}(t) e^{i m \boldsymbol{\theta}}, i e^{i m \theta_{k}}\right\rangle, \quad K<0
$$

even when $L(t)$ is nonsymmetric or time-varying. The dynamics (12) linearize to (11) in the neighborhood of a synchronized state, showing that local synchronization is indeed achieved by the dynamical system (12) under the sole assumption that $G(t)$ is uniformly connected. A stronger convergence result is proven in [16] by mapping the dynamics (12) into the Euclidean space. This change of coordinates requires restricting the set of initial phases in an open interval $\left(\theta_{0}-\pi / 2, \theta_{0}+\pi / 2\right)$. An alternative proof is provided in [21]. For initial conditions in a broader interval, simulation results indicate that, in contrast with the consensus dynamics (11), synchronization is not achieved in general by the dynamical system (12). This fact is not surprising when considering that the proof of Proposition 1 rests on a contraction property for the convex hull of the $N$ states $x_{k} \in \mathbb{C}$, a proof concept that does not extend to the torus.

Comparing the dynamics (12) to the (all-to-all) gradient dynamics (5), one is led to interpret $\sum_{l \sim k} e^{i m \theta_{l}} / d_{\max }$ as a (local) static estimate of the average quantity $\sum_{l \neq k} e^{i m \theta_{l}} / N-1$. In contrast to the consensus dynamics (11), the use of this static estimate in (12) fails to recover the global convergence results of the gradient dynamics (5). The approach recently proposed in [24] shows that the global convergence results can be recovered by using a local dynamic estimate instead. We summarize the main results of [24] in the following theorems. The reader is referred to [24] for detailed proofs.

Theorem 3 (dynamic synchronization): Let $G(t)$ be a uniformly connected $\delta$-digraph and $L(t)$ the corresponding Laplacian matrix. Consider the phase model $\dot{\boldsymbol{\theta}}=\boldsymbol{u}$. Then, the (dynamic) control

$$
\begin{aligned}
& u_{k}=-\frac{K}{m}\left\langle w_{k}, i\right\rangle, \quad K<0 \\
& \dot{w}_{k}=i K w_{k}\left\langle w_{k}, i\right\rangle+\sum_{j=1}^{N} a_{k j}(t) w_{j} e^{i m\left(\theta_{j}-\theta_{k}\right)}
\end{aligned}
$$

enforces exponential convergence of solutions $\theta(t)$ to the critical set of $U_{m}$. Only the set of synchronized states modulo $2 \pi / m$ is asymptotically stable and every other limit set is unstable. Furthermore, if $G(t)$ is a balanced digraph and if $w_{k}(0)=1$ for each $k$, then the asymptotic consensus value for $e^{i m \theta_{k}} / m$, for $k=$ $1, \ldots, N$, is the average $p_{m \theta}(0)=(1 /(m N)) \sum_{k=1}^{N} e^{i m \theta_{k}(0)}$.

Proof: Set $x_{k}=w_{k} e^{i m \theta_{k}} / m$. Then, $\boldsymbol{x}$ obeys the consensus dynamics $\dot{\boldsymbol{x}}=-L(t) \boldsymbol{x}$, which implies that the solution $\boldsymbol{x}(t)$ exponentially converges to the consensus variable $\bar{x} \mathbf{1}$. This implies that the dynamics $\dot{\theta}_{k}=-(K / m)\left\langle w_{k}, i\right\rangle$ exponentially converges to the (time-invariant) dynamics $\dot{\theta}_{k}=-K$ $\left\langle\mid x, i e^{i m \theta_{k}}\right\rangle$. Consequently, $\boldsymbol{\theta}(t)$ asymptotically converges to an equilibrium in the critical set of $U_{m}$, and only the set of synchronized states is asymptotically stable. If $G(t)$ is balanced, then $\langle\mathbf{1}, \boldsymbol{x}\rangle$ is a conserved quantity and $\bar{x}=$ $(1 /(m N)) \sum_{k=1}^{N} w_{k}(0) e^{i m \theta_{k}(0)}=p_{m \theta}(0)$.

Theorem 4 (dynamic balancing): Let $G(t)$ be a uniformly connected balanced $\delta$-digraph and $L(t)$ the corresponding Laplacian matrix. Consider the phase model $\dot{\boldsymbol{\theta}}=\boldsymbol{u}$. If $w_{k}(0)=1$ for each $k$, then the (dynamic) control

$$
\begin{aligned}
& u_{k}=-\frac{K}{m}\left\langle w_{k}, i\right\rangle, \quad K>0 \\
& \dot{w}_{k}=i K\left(w_{k}-1\right)\left\langle w_{k}, i\right\rangle+\sum_{j=1}^{N} a_{k j}(t) w_{j} e^{i m\left(\theta_{j}-\theta_{k}\right)}
\end{aligned}
$$

enforces exponential convergence of solutions $\theta(t)$ to the critical set of $U_{m}$. Furthermore, only the set of balanced states modulo $2 \pi / m$ is asymptotically stable and every other limit set is unstable.

Proof: Set $x_{k}=w_{k} e^{i m \theta_{k}} / m$. Then, $\boldsymbol{x}$ satisfies the dynamics

$$
\dot{\boldsymbol{x}}=-L(t) \boldsymbol{x}+i \operatorname{diag}\left\{u_{k}\right\} e^{i m \boldsymbol{\theta}}=-L(t) \boldsymbol{x}+\frac{d}{d t} \frac{e^{i m \boldsymbol{\theta}}}{m} .
$$

As a consequence, the quadratic form $V(\boldsymbol{x})=(1 / 2)\langle\boldsymbol{x}, \boldsymbol{x}\rangle$ is nonincreasing along the solutions and satisfies

$$
\begin{aligned}
\dot{V} & =-\langle\boldsymbol{x}, L(t) \boldsymbol{x}\rangle+\sum_{k=1}^{N} u_{k}\left\langle x_{k}, i e^{i m \theta_{k}}\right\rangle \\
& =-\langle\boldsymbol{x}, L(t) \boldsymbol{x}\rangle-\frac{1}{K} \sum_{k=1}^{N} u_{k}^{2} \leq 0
\end{aligned}
$$

We deduce from (16) that $u_{k}$ is a time function in $\mathcal{L}_{2}(0,+\infty)$ along each solution of the closed-loop dynamics. But, then (15) is the consensus dynamics (11) with an additive perturbation in $\mathcal{L}_{2}(0,+\infty)$, which does not destroy the convergence of $\boldsymbol{x}(t)$ to a consensus equilibrium $\mathbf{1} \bar{x}$. Since $G(t)$ is balanced, one obtains from (15)

$$
\frac{1}{N}\langle\mathbf{1}, \dot{\boldsymbol{x}}\rangle=\frac{1}{N}\left\langle\mathbf{1}, \frac{d}{d t} \frac{e^{i m \boldsymbol{\theta}}}{m}\right\rangle .
$$

Integrating both sides of (17) and using the fact that $x_{k}(0)=$ $e^{i m \theta_{k}(0)} / m$, one concludes that $(1 / N) \sum_{k=1}^{N} x_{k}(t)=p_{m \theta}(t)$ for all $t \geq 0$. This implies that the dynamics $\dot{\theta}_{k}=-(\mathrm{K} / \mathrm{m})$ $\left\langle w_{k}, i\right\rangle$ asymptotically converges to the (time-invariant) dynamics $\dot{\theta}_{k}=-K\left\langle p_{m \theta}, i e^{i m \theta_{k}}\right\rangle$. This shows that $\theta(t)$ asymptotically converges to an equilibrium in the critical set of $U_{m}$ and that only the set of balanced states modulo $2 \pi / m$ is asymptotically stable.

Theorems 3 and 4 generalize the global convergence results of the all-to-all gradient control (5) under weak assumptions on the communication graph. This generalization is obtained at the price of increased communication between the communicating particles because particles must not only exchange their relative configuration variables, but also their local estimate of the average $p_{m \theta}$. In both theorems, the variable $w_{k}$ can be interpreted as a local estimate of $p_{m \theta}$ in a reference frame attached to particle $k$, while $x_{k}=w_{k} e^{i m \theta_{k}} / m$ is the local estimate of $p_{m \theta}$ in 


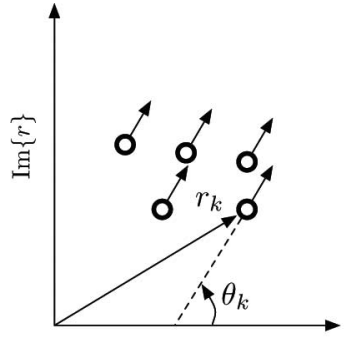

$\operatorname{Re}\{r\}$

(a)

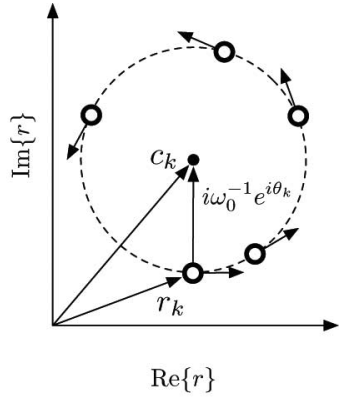

(b)
Fig. 2. Synchronizing the state variables $s_{k}=-i \omega_{0} c_{k}$. (a) If $\omega_{0}=0$, then synchronization of $s_{k}$ generates a parallel formation. (b) If $\omega_{0} \neq 0$, then synchronization of $s_{k}$ generates a circular formation centered at $c_{k}=$ $r_{k}+i \omega_{0}^{-1} e^{i \theta_{k}}$.

an absolute reference frame. Consensus filters that reconstruct possibly time-varying average quantities have been recently proposed in [25]. It should be noted that the balancing control law in Theorem 4 requires proper initialization of the estimator variables. This is a potential limitation of the consensus algorithm in applications that involve network reconfigurations such as a time-varying number of agents. Such limitations could possibly be alleviated by exploiting the recent work [31] on consensus algorithms.

All of the convergence results in this section still hold when adding a constant $\omega_{0} \in \mathbb{R}$ to $u_{k}$. The constant $\omega_{0}$ is eliminated from the equations by using the change of coordinates $\tilde{\boldsymbol{\theta}}=\boldsymbol{\theta}-\omega_{0} t \mathbf{1}$, which amounts to expressing the dynamics of $\boldsymbol{\theta}$ in a rotating frame. The feedback control laws are invariant under this change of coordinates because they are expressed in the relative coordinates $\theta_{k}-\theta_{j}$. In geometric terms, the stabilization takes place in the shape manifold $T^{N} / S^{1}$. We exploit this feature in the subsequent sections.

\section{Stabilization of Parallel and Circular Formations}

A basic observation in the companion paper [11] is to reformulate the stabilization of parallel and circular formations in the collective model (1) as the task of synchronizing the state variables

$$
s_{k}=-i \omega_{0} c_{k}=e^{i \theta_{k}}-i \omega_{0} r_{k}, \quad k=1, \ldots, N .
$$

An illustration is provided in Fig. 2: for $\omega_{0}=0$, the synchronization of $s_{k}$ is equivalent to the synchronization of the phase variables $\theta_{k}$. This corresponds to a parallel formation in the collective model (1). For $\omega_{0} \neq 0$, the state $c_{k}=i s_{k} / \omega_{0}$ is the center of the circle of radius $\rho_{0}=\left|\omega_{0}\right|^{-1}$ traversed by particle $k$ under the action of the constant control $u_{k}=\omega_{0}$. A circular relative equilibrium is obtained when all the centers coincide, which corresponds to synchronization of the variables $s_{k}$.

\section{A. Time-Invariant and Undirected Communication Graphs}

In our previous paper [11], synchronization of the state variables $s_{k}$ defined in (18) is achieved by minimizing the quadratic form $\|P s\|^{2}=\langle s, P s\rangle$, which, for $\omega_{0}=0$, reduces to the phase synchrony measure (6). Viewing $P$ as $1 / N$ times the Laplacian of a complete graph, one is led to the following extension.

Theorem 5 (Laplacian control): Let $G$ be a connected and balanced graph and $L$ be the Laplacian of $G$. Then, the quadratic potential $Q_{L}(s)=(1 / 2) d_{\max }\langle s, L s\rangle$ reaches its global minimum in the set of synchronized states $s=1 s_{0}$. If $G$ is undirected, this set is (locally) exponentially stabilized by the control law

$$
u_{k}=\omega_{0}+\frac{K}{d_{\max }}\left\langle L_{k} s, i e^{i \theta_{k}}\right\rangle, \quad K<0 .
$$

If $\omega_{0} \neq 0$, then the convergence is global.

Proof: The proof is entirely analogous to the proof of Theorem 2 in the companion paper [11] except that the all-to-all Laplacian $(N) P$ is replaced by the Laplacian $L$ of an arbitrary connected and undirected graph. If $\omega_{0} \neq 0$, then $s$ takes values in $\mathbb{C}^{N}$, and the convergence is global because the quadratic form $Q(s)$ has no other minima. If $\omega_{0}=0$, then the control (19) reduces to (12), and the result is only local, except for complete graphs.

\section{B. Uniformly Connected Communication Graphs}

For a complete graph, the control law (19) with $K=$ $\left(d_{\max } /\left(d_{\max }+1\right)\right) \tilde{K}$ takes the form

$$
u_{k}=\omega_{0}+\tilde{K}\left\langle s_{k}-\left(p_{\theta}-i \omega_{0} R\right), i e^{i \theta_{k}}\right\rangle, \quad \tilde{K}<0,
$$

where the position $R=(1 / N) \sum_{k=1}^{N} r_{k}$ and the velocity $p_{\theta}=$ $(1 / N) \sum_{k=1}^{N} e^{i \theta_{k}}$ are quantities averaged over the entire group. In a manner analogous to the result of Section III-C, these average quantities can be estimated by a consensus filter, leading to a generalization of the control law of Theorem 5 to arbitrary communication graphs.

Theorem 6 (dynamic Laplacian control): Let $G(t)$ be a uniformly connected $\delta$-digraph and $L(t)$ the corresponding Laplacian matrix. Then, the (dynamic) control

$$
\begin{aligned}
& u_{k}=\omega_{0}-K\left\langle w_{k}-i \omega_{0} v_{k}, i\right\rangle, \quad K<0 \\
& \dot{w}_{k}=i w_{k} u_{k}+\sum_{j=1}^{N} a_{k j}(t) w_{j} e^{i\left(\theta_{j}-\theta_{k}\right)} \\
& \dot{v}_{k}=-1-i v_{k} u_{k}+\sum_{j=1}^{N} a_{k j}(t)\left(v_{j} e^{i\left(\theta_{j}-\theta_{k}\right)}+r_{k}-r_{j} e^{-i \theta_{k}}\right)
\end{aligned}
$$

enforces exponential convergence of solutions to a relative equilibrium of the collective model (1). If $\omega_{0}=0$, the relative equilibrium is a parallel formation. For $\omega_{0} \neq 0$, the relative equilibrium is a circular formation of radius $\rho_{0}=$ $\left|\omega_{0}\right|^{-1}$ with direction of rotation determined by the sign of $\omega_{0}$.

Proof: Set $x_{k}=w_{k} e^{i \theta_{k}}$ and $y_{k}=v_{k} e^{i \theta_{k}}+r_{k}$. Then, $\boldsymbol{x}$ and $\boldsymbol{y}$ obey the consensus dynamics $\dot{\boldsymbol{x}}=-L(t) \boldsymbol{x}$ and $\dot{\boldsymbol{y}}=$ $-L(t) \boldsymbol{y}$, respectively, which implies that the solutions $\boldsymbol{x}(t)$ and $\boldsymbol{y}(t)$ exponentially converge to the consensus variables $\bar{x} \mathbf{1}$ and $\bar{y} \mathbf{1}$, respectively. This implies that the dynamics $\dot{\theta}_{k}=u_{k}$ 
exponentially converge to the (time-invariant) dynamics

$$
\dot{\theta}_{k}=\omega_{0}+K\left\langle s_{k}-\left(\bar{x}-i \omega_{0} \bar{y}\right), i e^{i \theta_{k}}\right\rangle .
$$

For $\omega_{0}=0,(22)$ is identical to the dynamic synchronization result of Theorem 3. For $\omega_{0} \neq 0$, the quadratic form

$$
S_{k}=\frac{1}{2}\left|s_{k}-\bar{x}+i \omega_{0} \bar{y}\right|^{2}
$$

satisfies $\dot{S}_{k}=K\left\langle s_{k}-\bar{x}+i \omega_{0} \bar{y}, i e^{i \theta_{k}}\right\rangle^{2} \leq 0$ along the solutions of (22). One concludes that all solutions converge to a circular formation of radius $\rho_{0}=\left|\omega_{0}\right|^{-1}$ centered at $c_{0}=$ $i \bar{x} / \omega_{0}+\bar{y}$.

In Theorem 6, the variable $w_{k}$ can be interpreted as a local estimate of $p_{\theta}$ in a reference frame attached to particle $k$, while $x_{k}=w_{k} e^{i \theta_{k}}$ is the local estimate of $p_{\theta}$ in an absolute reference frame. The variable $v_{k}$ can be interpreted as a local estimate of the center of mass $R$ in a reference frame attached to particle $k$, while $y_{k}=v_{k} e^{i \theta_{k}}+r_{k}$ is the local estimate of $R$ in an absolute reference frame.

\section{Stabilization of Symmetric Patterns}

An interesting feature of the phase potentials derived in Section III and the spacing potentials derived in Section IV is that they can be combined additively. The phase potentials $W_{m}, 1 \leq m \in \mathbb{N}$, defined in (7) satisfy

$$
\dot{W}_{m}=\sum_{k=1}^{N} \frac{\partial W_{m}}{\partial \theta_{k}} u_{k}
$$

and, because they are invariant to a rigid rotation $\theta \mapsto \theta+\omega_{0} \mathbf{1}$, they also satisfy

$$
\dot{W}_{m}=\sum_{k=1}^{N} \frac{\partial W_{m}}{\partial \theta_{k}}\left(u_{k}-\omega_{0}\right)
$$

for an arbitrary $\omega_{0} \in \mathbb{R}$. Likewise, the spacing potential $Q_{L}(\boldsymbol{s})$ defined in Theorem 5 satisfies

$$
\dot{Q}_{L}=\frac{1}{d_{\max }} \sum_{k=1}^{N}\left\langle L_{k} \boldsymbol{s}, i e^{i \theta_{k}}\right\rangle\left(u_{k}-\omega_{0}\right) .
$$

As a consequence, a linear combination of the potentials

$$
V(\boldsymbol{s}, \boldsymbol{\theta})=-K Q_{L}(\boldsymbol{s})-\sum_{m=1}^{M} K_{m} W_{m}(\boldsymbol{\theta}), \quad K<0
$$

satisfies

$$
\dot{V}=-\sum_{k=1}^{N}\left(\frac{K}{d_{\max }}\left\langle L_{k} \boldsymbol{s}, i e^{i \theta_{k}}\right\rangle+\sum_{m=1}^{M} K_{m} \frac{\partial W_{m}}{\partial \theta_{k}}\right)\left(u_{k}-\omega_{0}\right) .
$$

The corresponding gradient-like control

$$
u_{k}=\omega_{0}+\frac{K}{d_{\max }}\left\langle L_{k} \boldsymbol{s}, i e^{i \theta_{k}}\right\rangle+\sum_{m=1}^{M} K_{m} \frac{\partial W_{m}}{\partial \theta_{k}}
$$

is thus the same linear combination of the individual gradientlike controls. A critical point that jointly minimizes the individual potentials $-K Q_{L}$ and $-K_{m} W_{m}, 1 \leq m \leq M$, also minimizes the Lyapunov function $V$. This suggests a versatile design method for the decentralized stabilization of relative equilibria that jointly minimize various combinations of the potentials proposed in the previous sections.

\section{A. Stabilization of Symmetric Balanced Patterns}

Let $1 \leq M \leq N$ be a divisor of $N$. An $(M, N)$-pattern is a symmetric arrangement of $N$ phases consisting of $M$ clusters uniformly spaced around the unit circle, each with $N / M$ synchronized phases. For any $N$, there exist, at least, two symmetric patterns: the $(1, N)$-pattern, which is the synchronized state, and the $(N, N)$-pattern, which is the so-called splay state, characterized by $N$ phases uniformly spaced around the circle. An $(M, N)$-pattern circular formation of radius $\rho_{0}$ is an isolated relative equilibrium of the collective model (1).

Lemma 2: Let $L$ be the Laplacian of a $d_{0}$-circulant graph with $N$ vertices. For $1 \leq m \in \mathbb{N}$, each $(M, N)$-pattern is a critical point of the potentials $W_{m}$ defined in (7).

Proof: Modulo a uniform rotation and with the notations of Lemma 1, an $(M, N)$-pattern is characterized by the phase arrangement

$$
\overline{\boldsymbol{\theta}}=\frac{N}{M} \phi
$$

This means that the vector $e^{i m \overline{\boldsymbol{\theta}}}=e^{i m(N / M) \phi}$ is the $k$ th eigenvector of $L$, with $k=1+(m(N / M)-1) \bmod N$. But, if $e^{i m \overline{\boldsymbol{\theta}}}$ is an eigenvector of $L$, then $\overline{\boldsymbol{\theta}}$ is a critical point of $\left\langle e^{i m \overline{\boldsymbol{\theta}}}, L e^{i m \overline{\boldsymbol{\theta}}}\right\rangle$, which proves the claim.

The following result was proven in [11] for complete graphs. An early version of the theorem for $d_{0}$-circulant graphs appeared in [13].

Theorem 7 (Laplacian stabilization of $(M, N)$-pattern circular formations): Consider a connected and $d_{0}$-circulant graph $G$, the associated Laplacian $L$, and the associated phase potentials $W_{m}(\boldsymbol{\theta})=(1 / 2) m^{2} d_{\max }\left\langle e^{i m \boldsymbol{\theta}}, \operatorname{Le} e^{i m \boldsymbol{\theta}}\right\rangle$. Let $1 \leq M \leq N$ be a divisor of $N$. If $\overline{\boldsymbol{\theta}} \in T^{N}$ is an $(M, N)$-pattern then it is a (local) minimum of the potential

$$
W_{L}^{M, N}=-\sum_{m=1}^{M} K_{m} W_{m}
$$

with $K_{m}>0$ for $m=1, \ldots, M-1$ and

$$
K_{M}<-\sum_{m=1}^{M-1} K_{m}>0 .
$$

An $(M, N)$-pattern circular formation of radius $\rho_{0}=\left|\omega_{0}\right|^{-1}$ is (locally) exponentially stabilized by the control law

$$
u_{k}=\omega_{0}+\frac{K}{d_{\max }}\left\langle L_{k} \boldsymbol{s}, i e^{i \theta_{k}}\right\rangle+\sum_{m=1}^{M} K_{m} \frac{\partial W_{m}}{\partial \theta_{k}} .
$$

Proof: By Lemma 2, $\overline{\boldsymbol{\theta}}$ is a critical point of $W_{L}^{M, N}$. We first show that $\overline{\boldsymbol{\theta}}$ is exponentially stabilized in the reduced space of relative phases by the gradient control

$$
\boldsymbol{u}=\sum_{m=1}^{M} K_{m} \frac{\partial W_{m}}{\partial \boldsymbol{\theta}}
$$


The Jacobian linearization of (28) about $\overline{\boldsymbol{\theta}}$ is a square matrix $\hat{L}$ that has entries

$$
\hat{l}_{k k}=\sum_{l=1}^{N} \frac{a_{k l}}{d_{0}} \sum_{m=1}^{M} K_{m}\left\langle e^{i m \bar{\theta}_{k}}, e^{i m \bar{\theta}_{l}}\right\rangle
$$

and, for $j \neq k$

$$
\begin{aligned}
\hat{l}_{k j} & =-\frac{a_{k j}}{d_{0}} \sum_{m=1}^{M} K_{m}\left\langle e^{i m \bar{\theta}_{k}}, e^{i m \bar{\theta}_{j}}\right\rangle \\
& =-\frac{a_{k j}}{d_{0}}\left(K_{M}+\sum_{m=1}^{M-1} K_{m}\left\langle e^{i m \bar{\theta}_{k}}, e^{i m \bar{\theta}_{j}}\right\rangle\right) .
\end{aligned}
$$

Using the condition (26) shows that $\hat{l}_{k j} \geq 0$ and $\hat{l}_{k j}=0$ if and only if $a_{k j}=0$. This implies that $-\hat{L}$ is the Laplacian of a graph $\hat{G}$ that, other than having different edge weights, is identical to $G$. Therefore, none of the eigenvalues of $\hat{L}$ are positive, and since $\hat{G}$ is connected, zero as an eigenvalue of $\hat{L}$ is simple.

The proof of the second part of Theorem 7 is a straightforward adaptation of [11, Th. 3]. Under the control (27), the Lyapunov function $V(\boldsymbol{s}, \boldsymbol{\theta})$ in (24) satisfies $\dot{V}=-\sum_{k=1}^{N}\left(u_{k}-\omega_{0}\right)^{2} \leq 0$ along the closed-loop solutions. By the LaSalle Invariance principle, solutions for the reduced system on shape space converge to the largest invariant set $\Lambda$ where

$$
\frac{K}{d_{0}}\left\langle L_{k} \boldsymbol{s}, i e^{i \theta_{k}}\right\rangle=-\sum_{m=1}^{M} K_{m} \frac{\partial W_{m}}{\partial \theta_{k}}
$$

for $k=1, \ldots, N$. In the set $\Lambda$, the dynamics reduce to $\dot{\theta}_{k}=\omega_{0}$, which implies that $W_{L}^{M, N}$ is constant. Therefore, the righthand side of (29) vanishes in the set $\Lambda$, which implies $L s=0$ since $\dot{s}=0$. We conclude that solutions converge to an $(M, N)$ pattern circular formation of radius $\rho_{0}=\left|\omega_{0}\right|^{-1}$.

Theorem 7 does not exclude convergence to circular formations that correspond to other critical points of the phase potential $W_{L}^{M, N}$. Simulations indicate that the basin of attraction of certain symmetric patterns is indeed small in the case of incomplete graphs. In contrast, convergence to a different critical point is rarely observed in simulations for a complete graph, suggesting a large basin of attraction in the case of all-to-all communication. The all-to-all control law makes use of the averaged quantities $p_{m \theta}, 1 \leq m \leq M$, and $(1 / N) \sum_{k=1}^{N} s_{k}$. For general graphs, these averaged quantities can be reconstructed by consensus filters so that the all-to-all result is recovered with the following dynamic control scheme.

Theorem 8 (dynamic stabilization of $(M, N)$-pattern circular formations): Let $G(t)$ be a balanced and uniformly connected $\delta$-digraph and $L(t)$ the corresponding Laplacian. Consider the collective model (1). Then, the set of $(M, N)$-pattern circular formations is (locally) exponentially stabilized by the (dynamic) control

$$
u_{k}=\omega_{0}-\left\langle K\left(w_{k}-i \omega_{0} v_{k}\right)+\sum_{m=1}^{M} \frac{K_{m}}{m} w_{k}^{(m)}, i\right\rangle
$$

$$
\begin{aligned}
+\dot{w}_{k}= & -i w_{k} u_{k}+\sum_{j=1}^{N} a_{k j}(t) w_{j} e^{i\left(\theta_{j}-\theta_{k}\right)} \\
+\dot{v}_{k}= & -1-i v_{k} u_{k}+\sum_{j=1}^{N} a_{k j}(t)\left(v_{j} e^{i\left(\theta_{j}-\theta_{k}\right)}+r_{k}-r_{j} e^{-i \theta_{k}}\right) \\
\dot{w}_{k}^{(m)}= & -i m\left(w_{k}^{(m)}-1\right)\left(u_{k}-\omega_{0}\right) \\
& +\sum_{j=1}^{N} a_{k j}(t) w_{j}^{(m)} e^{i m\left(\theta_{j}-\theta_{k}\right)} \\
+\dot{w}_{k}^{(M)}= & -i M w_{k}^{(M)}\left(u_{k}-\omega_{0}\right)+\sum_{j=1}^{N} a_{k j}(t) w_{j}^{(M)} e^{i m\left(\theta_{j}-\theta_{k}\right)}
\end{aligned}
$$

with gains $K<0, K_{m}>0, m=1, \ldots, M-1$, and $K_{M}<0$ and provided the initial conditions satisfy $w_{k}(0)=1, v_{k}(0)=$ $0, w_{k}^{(M)}(0)=1$, and $w_{k}^{(m)}(0)=1$.

Proof: Set $x_{k}=w_{k} e^{i \theta_{k}}$ and $y_{k}=v_{k} e^{i \theta_{k}}+r_{k}$. Then, $\boldsymbol{x}$ and $\boldsymbol{y}$ obey the consensus dynamics $\dot{\boldsymbol{x}}=-L(t) \boldsymbol{x}$ and $\dot{\boldsymbol{y}}=-L(t) \boldsymbol{y}$, respectively, which implies that the solutions $\boldsymbol{x}(t)$ and $\boldsymbol{y}(t)$ exponentially converge to the consensus variables $\bar{x} \mathbf{1}$ and $\bar{y} \mathbf{1}$, respectively. Similarly, set $x_{k}^{(M)}=w_{k}^{(M)} e^{i M\left(\theta_{k}-\omega_{0} t\right)} / M$, then

$$
\dot{\boldsymbol{x}}^{(M)}=-L(t) \boldsymbol{x}^{(M)}
$$

which implies that $\boldsymbol{x}^{(M)}(t)$ converges to consensus variables $\bar{x}^{(M)} \mathbf{1}$.

Next, we prove exponential stability of $(M, N)$-pattern circular formations with the modified control

$$
\begin{aligned}
\tilde{u}_{k} & =\omega_{0}+K\left\langle s_{k}-\left(\bar{x}-i \omega_{0} \bar{y}\right), i e^{i \theta_{k}}\right\rangle \\
& -\sum_{m=1}^{M-1} \frac{K_{m}}{m}\left\langle w_{k}^{(m)}, i\right\rangle-\frac{K_{M}}{M}\left\langle\bar{x}^{(M)}, i e^{i M\left(\theta_{k}-\omega_{0} t\right)}\right\rangle .
\end{aligned}
$$

Because the closed-loop systems obtained with the control laws $u_{k}$ and $\tilde{u}_{k}$ only differ by a perturbation in $\mathcal{L}_{2}[0, \infty)$, exponential stability of the modified system implies convergence of the solutions to the equilibrium in the original system as well. Set $x_{k}^{(m)}=w_{k}^{(m)} e^{i m\left(\theta_{k}-\omega_{0} t\right)} / m, m=1, \ldots, M-1$, then $\boldsymbol{x}^{(m)}(t)$ obeys the dynamics

$$
\begin{aligned}
\dot{\boldsymbol{x}}^{(m)} & =-L(t) \boldsymbol{x}^{(m)}+i \operatorname{diag}\left\{u_{k}-\omega_{0}\right\} e^{i m\left(\boldsymbol{\theta}-\omega_{0} t \mathbf{1}\right)} \\
& =-L(t) \boldsymbol{x}^{(m)}+\frac{d}{d t}\left(\frac{e^{i m\left(\boldsymbol{\theta}-\omega_{0} t \mathbf{1}\right)}}{m}\right), m=1, \ldots, M-1 .
\end{aligned}
$$

Consider the Lyapunov function $V=-K \sum_{k=1}^{N} S_{k}+$ $(1 / 2) \sum_{m=1}^{M-1} K_{m}\left\|\boldsymbol{x}^{(m)}\right\|^{2}-\left(K_{M} / 2\right) \| e^{i M\left(\boldsymbol{\theta}-\omega_{0} t \mathbf{1}\right)} / M-\bar{x}^{(M)}$ $\mathbf{1} \|^{2}$ with $S_{k}$ defined by (23). With the control law (31), $V$ is nonincreasing along the solutions and satisfies

$$
\dot{V}=-\sum_{m=1}^{M-1} K_{m}\left\langle\boldsymbol{x}^{(m)}, L(t) \boldsymbol{x}^{(m)}\right\rangle-\sum_{k=1}^{N}\left(\tilde{u}_{k}-\omega_{0}\right)^{2} \leq 0 .
$$


This implies that $\tilde{u}_{k}$ is a time function in $\mathcal{L}_{2}(0,+\infty)$ along each solution of the closed-loop dynamics. For each $m \in$ $\{1, M-1\},(32)$ is a linear exponentially stable system with an additive perturbation in $\mathcal{L}_{2}(0,+\infty)$. This implies that each estimator $\boldsymbol{x}^{(m)}(t), 1 \leq m \leq M-1$, converges to a consensus equilibrium $\mathbf{1} \bar{x}^{(m)}$. Since $\bar{G}(t)$ is balanced, one obtains from (32)

$$
\frac{1}{N}\left\langle\mathbf{1}, \dot{\boldsymbol{x}}^{(m)}\right\rangle=\frac{1}{N}\left\langle\mathbf{1}, \frac{d}{d t} \frac{e^{i m\left(\boldsymbol{\theta}-\omega_{0} t \mathbf{1}\right)}}{m}\right\rangle
$$

for $m=1, \ldots, M-1$. Integrating both sides of (33) and using the fact that $\boldsymbol{x}^{(m)}(0)=e^{i m \boldsymbol{\theta}(0)} / m$, one concludes that $(1 / N) \sum_{k=1}^{N} x_{k}^{(m)}(t)=e^{-i m \omega_{0} t} p_{m \theta}(t)$ for all $t \geq 0$. Using that each $x_{k}^{(m)}(t)$ converges to $e^{-i m \omega_{0} t} p_{m \theta}(t), 1 \leq m \leq M$, one concludes that, along each solution of the closed-loop system, the dynamics $\dot{\theta}_{k}$ asymptotically converge to the (all-to-all) control law

$$
\begin{aligned}
\bar{u}_{k}= & \omega_{0}+K\left\langle s_{k}-\bar{x}+i \omega_{0} \bar{y}, i e^{i \theta_{k}}\right\rangle \\
& -\sum_{m=1}^{M} K_{m}\left\langle p_{m \theta}, i e^{i \theta_{k}}\right\rangle .
\end{aligned}
$$

As a consequence, the only limit sets of the closed-loop dynamics are circular formations with a phase arrangement in the critical set of the (all-to-all) potential $W_{N P}^{(M, N)}$. In particular, the $(M, N)$-pattern circular formation is an exponentially stable equilibrium of the closed-loop system.

Exponential stabilization of $(M, N)$-pattern circular formations was considered in our previous paper [11] in the framework of complete graphs (all-to-all communication). Theorem 8 extends that result to the framework of general communication graphs. As mentioned in our earlier paper, the result is only local, but simulations suggest a large region of attraction.

\section{SYMMETRY BREAKING}

All the control laws discussed so far are control laws in the shape space, that is, the closed-loop dynamics are invariant to rigid translations and rotations in the plane, which corresponds to the action of the symmetry group $S E(2)$. It is of interest, in some applications, to break this $S E(2)$ symmetry. For phase synchronization, this means forcing the synchronization to a specific phase $\theta_{0} \in S^{1}$. For the control of circular formation, this means forcing the formation to a specific circle in the plane. We show in this section how these can be achieved in a decentralized way.

Let the reference frame be described by the (virtual) reference particle with dynamics $\dot{r}_{0}=e^{i \theta_{0}}, \dot{\theta}_{0}=\omega_{0}$. If $\omega_{0}=0, \theta_{0}$ serves as a fixed reference orientation. If $\omega_{0} \neq 0$, then the reference particle traces a circle of radius $\rho_{0}=\left|\omega_{0}\right|^{-1}$ centered at $c_{0}=$ $r_{0}+i \omega_{0}^{-1} e^{i \theta_{0}}=: i s_{0} / \omega_{0}$. In that case, $\theta_{0}(t)$ serves as reference orientation and $c_{0}$ serves as a reference center for the circular formation.

In order to describe the information flow from the (virtual) reference particle to the particles $k$, we augment the communication $\delta$-digraph $G(t)$ by an additional reference node 0 , with directed edges from 0 to the informed particles. The augmented $\delta$-digraph is denoted by $\underset{\tilde{G}}{\tilde{G}}(t)$ and its Laplacian matrix is denoted by $\tilde{L}(t)$. The Laplacian $\tilde{L}(t)$ has the block structure

$$
\tilde{L}(t)=\left[\begin{array}{c|c}
0 & 0 \ldots 0 \\
\hline l_{10}(t) & \\
\vdots & L(t)-\operatorname{diag}\left\{l_{k 0}(t)\right\} \\
l_{N 0}(t) &
\end{array}\right]
$$

Note that, in order for the uniform connectedness assumption to be satisfied for the extended $\delta$-digraph $\tilde{G}(t)$, there must be a path from reference node 0 to each of the other nodes which respects the orientation of the edges of the graph.

\section{A. Time-Invariant and Undirected Communication Graphs}

For a fixed graph, the potential $S_{0}\left(s, s_{0}\right)=-\sum_{k=1}^{N} l_{k 0} \mid s_{k}-$ $\left.s_{0}\right|^{2}$ satisfies

$$
\dot{S}_{0}=-\sum_{k=1}^{N} l_{k 0}\left\langle s_{k}-s_{0}, i e^{i \theta_{k}}\right\rangle\left(u_{k}-\omega_{0}\right) .
$$

Augmenting the potential $Q_{L}(s)$ in Theorem 5 by the symmetry-breaking potential $S_{0}$ results in the augmented control law

$$
u_{k}=\omega_{0}+\frac{K}{d_{\max }}\left(\left\langle L_{k} s, i e^{i \theta_{k}}\right\rangle+l_{k 0}\left\langle s_{k}-s_{0}, i e^{i \theta_{k}}\right\rangle\right)
$$

with $K<0$. As a direct corollary of Theorem 5, the symmetrybreaking control law (35) stabilizes a parallel formation with orientation $\theta_{0}$, if $\omega_{0}=0$, and a circular formation centered at $c_{0}$, if $\omega_{0} \neq 0$. The same modification in Theorem 7 stabilizes an $(M, N)$-pattern circular formation centered at $s_{0}$, breaking the translation symmetry of the control law. The rotation symmetry can also be broken by synchronizing the orientation of the $(M, N)$-pattern with the reference $\theta_{0}(t)$. To this end, the phase potential $W_{L}^{M, N}(\boldsymbol{\theta})$ must be augmented by the symmetrybreaking potential $S_{0}\left(e^{i m \boldsymbol{\theta}}, e^{i M \theta_{0}}\right)$. The resulting control law is

$$
\begin{aligned}
u_{k}= & \omega_{0}+\frac{K}{d_{\max }}\left(\left\langle L_{k} s, i e^{i \theta_{k}}\right\rangle+l_{k 0}\left\langle s_{k}-s_{0}, i e^{i \theta_{k}}\right\rangle\right) \\
& +\sum_{m=1}^{M} K_{m} \frac{\partial W_{m}}{\partial \boldsymbol{\theta}}-\frac{K_{M}}{d_{\max }} l_{k 0}\left\langle e^{i M \theta_{0}}, i e^{i M \theta_{k}}\right\rangle .
\end{aligned}
$$

As a direct corollary of Theorem 7 , the symmetry-breaking control law (36) stabilizes an $(M, N)$-pattern circular formation centered at $c_{0}$ and with all phases synchronized modulo $2 \pi / M$ with the reference $\theta_{0}(t)$.

\section{B. Uniformly Connected Communication Graphs}

Symmetry breaking can also be achieved in the dynamic control algorithms of the previous sections. In order to break the translation symmetry in Theorem 6, one must simply consider the augmented graph $\tilde{G}(t)$ in the estimation dynamics of $w_{k}$ 
and $v_{k}$, that is

$$
\begin{aligned}
& \dot{w}_{k}=-i w_{k} u_{k}+\sum_{j=1}^{N} a_{k j}(t) w_{j} e^{i\left(\theta_{j}-\theta_{k}\right)} \\
& \dot{v}_{k}=-1-i v_{k} u_{k}+\sum_{j=1}^{N} a_{k j}(t)\left(v_{j} e^{i\left(\theta_{j}-\theta_{k}\right)}-r_{k}-r_{j} e^{-i \theta_{k}}\right)
\end{aligned}
$$

with the definition of the control law unchanged for $k=$ $1, \ldots, N$ and $u_{0}=\omega_{0}$. The consensus dynamics for $x_{k}$ $=w_{k} e^{i \theta_{k}}$ and $y_{k}=v_{k} e^{i \theta_{k}}+r_{k}$ are $\dot{\boldsymbol{x}}=-\tilde{L}(t) \boldsymbol{x}$ and $\dot{\boldsymbol{y}}=$ $-\tilde{L}(t) \boldsymbol{y}$, respectively. Because $\dot{x}_{0} \equiv 0$ and $\dot{y}_{0} \equiv 0$, the only possible consensus values are $x_{0}(0)$ and $y_{0}(0)$, respectively. Setting $w_{0}(0)=1$ and $v_{0}(0)=0$ results in $x(0)=e^{i \theta_{0}}$ and $y(0)=$ $r_{0}(0)$. If $\tilde{G}(t)$ is uniformly connected, the (modified) control law of Theorem 6 then achieves exponential convergence to a circular formation centered at $c_{0}=i \bar{x} \omega_{0}^{-1}+\bar{y}=r_{0}+i e^{i \theta_{0}} \omega_{0}^{-1}$, which breaks the translation symmetry of the formation.

A similar modification in the control law of Theorem 8 additionally enforces phase synchronization modulo $2 \pi / m$ to the reference $\theta_{0}(t)$, which also breaks the rotational symmetry of the phase arrangement.

\section{DESIGN OF COMMUNICATION GRAPHS AND APPLICATIONS}

In this section, we consider design of communication graphs and illustrate how design can be used to meet performance requirements in applications. Design of a communication graph for our many particle system refers to the choice of which of the available communication links should be used in the steering control law at any given time. In the case that the particle system models a multiagent system, availability of communication links depends upon the technology used for sensing and communication among particles. It may be advantageous for system performance, efficiency, and/or simplicity to make limited use of available communication. Further, different choices of communication graph for the different terms in the steering control law yield multiscale network patterns, as we describe later.

The development in this paper is strongly motivated by the application to mobile sensor networks used for autonomous ocean sampling [8], [9]. In this particular application, each agent in the network is an autonomous underwater glider, moving at near constant speed relative to the water, carrying sensors to measure the ocean (temperature, salinity, currents) and communicating only with a central on-shore computer (via Iridium satellite). Each agent can communicate at fairly regular intervals with the central computer; however, the particles do not synchronize their communication. In [32], a methodology is presented that enables control strategies for collective motion using the idealized particle model with decentralized steering control for planning, together with feedback provided via the asynchronous communication. Experimental results illustrate the methodology.

Because communication in this application is made through a central computer, there is, in principle, the opportunity to ap- proximate all-to-all communication, and therefore, to design any kind of limited communication graphs as appropriate. Justh and Krishnaprasad have applied their coordinated control strategies to an experimental network of unmanned aircraft. In this experiment, all-to-all communication is available, making it, therefore, also possible to design communication graphs as desired [33]. Even in applications where technology limits communication, there is always the opportunity to use less than is available, and this will be desirable if it can be done so to advantage.

In the ocean sampling network application and mobile sensor network applications, in general, the value of the collected measurement data can depend strongly on the collective motion pattern of the moving sensors. For instance, in the optimal mobile sensor coverage problem discussed in [9], patterns consisting of sensors moving around closed curves with coordinated phasing are practical solutions for maximizing information in data collected in a spatially and temporally varying field. Multiscale patterns become significant when there are multiple significant scales in the sampled region. For example, in the ocean sampling problem, it is of interest to sample well both the boundary of a region for fluxes and the interior of a region for dynamics. Smaller scales become important with the emerging presence of features such as an eddy or a jet.

There are many competing factors to consider in designing a communication graph for a given system. For example, the communication graph can influence the rate of convergence and region of attraction for stable patterns [18]. Fewer communication links may minimize the complexity of the network, but could increase the sensitivity to failures. As illustrated in [14], for the example of the ring graph topology, the choice of communication graph can also be used as a way to isolate desired patterns.

We propose a communication graph design concept, of particular relevance to sensor network applications, that uses multiple graphs at a time to yield multiscale (sensing) patterns. In this context, the different graphs are used in the different terms in the control law, so that subgroups can form and both intra- and intersubgroup coordination can be regulated. In the following, we present three examples to illustrate the design concept and versatility.

1) Separate spacing and phase graphs: We divide the $N$ particles into $B$ blocks (subgroups) of particles with $n=N / B$ particles in each subgroup. Although we assume that each subgroup is the same size in this example, this is not required. To each block, we assign an all-to-all graph with Laplacian $n P_{n}=n I_{n}-\mathbf{1 1}^{T}$. The block-all-to-all graph Laplacian is $L=I_{B} \otimes\left(n P_{n}\right)$, where $I_{B}=\operatorname{diag}\left\{\mathbf{1}_{B}\right\}$ and $\otimes$ is the Kronecker product. We assign $L$ to be the communication graph for the spacing control term so that in the case of circular control, particles within any subgroup move around the same circle, but circles associated with the different subgroups are independently located. For the phase control, we assign the all-to-all graph with Laplacian $N P$; this ignores subgroups and fixes the relative phases of all particles. Choosing the control

$$
u_{k}=\omega_{0}+\frac{K}{d_{\max }}\left\langle L_{k} \boldsymbol{s}, i e^{i \theta_{k}}\right\rangle-\frac{\partial W_{N P}^{M, N}}{\partial \theta_{k}}
$$




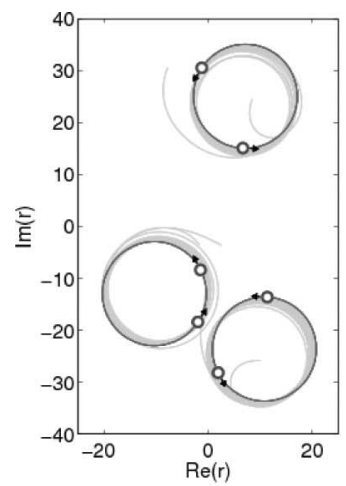

(a)

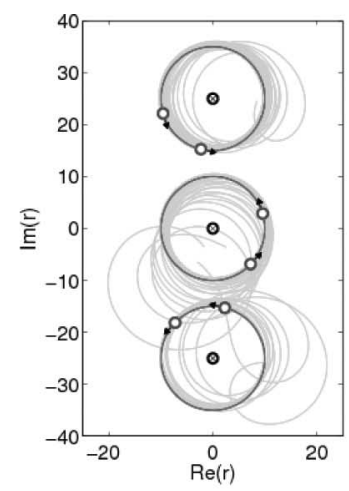

(b)

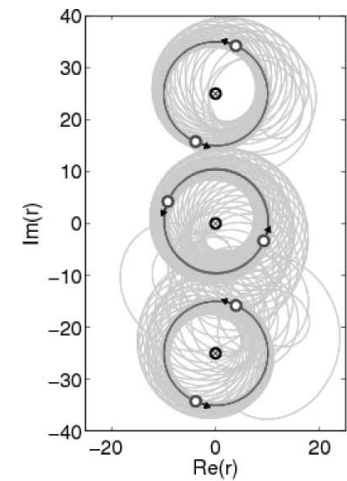

(c)

Fig. 3. Simulation results of (1) subject to Laplacian control with separate spacing and phase graphs with reference particles and nested phase potentials. (a) Control (38) with $M=N=6, B=3$, and $K=\omega_{0}=0.1$ stabilizes three separate circular formations of radius $\rho_{0}=\left|\omega_{0}^{-1}\right|$ and a splay arrangement of all 6 particle phases. (b) Control (39) fixes the centers of the circular formations to the reference locations $c_{0}^{(b)}=i 25(b-2), b=1,2,3$ denoted by crossed circles. (c) Control (40) stabilizes a nested $(\boldsymbol{M}, \boldsymbol{N})$-pattern with $Q=3$ and $\boldsymbol{M}=\boldsymbol{N}=(2,4,4)^{T}$. Trajectories start from random initial conditions, and are plotted after elapsed time equivalent to 50 revolutions; the last revolution of each particle is plotted in dark gray.

stabilizes an $(M, N)$-pattern over $B$ separate circular formations of radius $\rho_{0}=\left|\omega_{0}^{-1}\right|$. Simulations of (1) subject to (38) are shown in Fig. 3(a) for $M=N=6, B=3$, and $K=\omega_{0}=0.1$. The phase gains are $K_{1}=0.04$ and $\left|K_{m}\right|=0.01$ for $m=$ $2, \ldots, 6$. Note that the phases of the $N=6$ particles are in the splay state and the relative positions of the $B=3$ circles are arbitrary.

2) Separate spacing and phase graphs with reference particles: To each of the $B$ blocks, we associate one reference particle that traces out a circle of radius $\rho_{0}=\left|\omega_{0}^{-1}\right|$ centered at $c_{0}^{(b)}, b=1, \ldots, B$. The reference particle is connected to at least one particle in each block. To each block, we assign the augmented Laplacian denoted by $\widetilde{n P_{n}}$, using the methodology from Section VI. The augmented block-all-to-all Laplacian is $\tilde{L}=I_{B} \otimes\left(\widetilde{n P_{n}}\right)$. Let $\tilde{s}$ denote the corresponding augmented vector $s$ defined by

$$
\tilde{\boldsymbol{s}}=\left(-i \omega_{0} c_{0}^{(1)}, s_{1}, \ldots, s_{n},-i \omega_{0} c_{0}^{(2)}, s_{n+1}, \ldots\right)^{T} .
$$

Choosing the control

$$
u_{k}=\omega_{0}+\frac{K}{d_{\max }}\left\langle\tilde{L}_{k} \tilde{\boldsymbol{s}}, i e^{i \theta_{k}}\right\rangle-\frac{\partial W_{N P}^{M, N}}{\partial \theta_{k}}
$$

stabilizes an $(M, N)$-pattern over $B$ separate circular formations of radius $\rho_{0}=\left|\omega_{0}^{-1}\right|$ centered at $c_{0}^{(b)}, b=1, \ldots, B$. Simulations of (1) subject to (39) are shown in Fig. 3(b) for $c_{0}^{(b)}=i 25(b-2), b=1,2,3$. All other parameters are the same as in the previous example. It can be observed in this case that the phases are still in the splay state, but now the circles are positioned as desired.

3) Separate spacing and phase graphs with reference particles and nested phase potentials: Let $\boldsymbol{M}=\left(M_{1}, \ldots, M_{Q}\right)^{T}$ and $\boldsymbol{N}=\left(N_{1}, \ldots, N_{Q}\right)^{T}$, where $M_{q}$ is a divisor of $N_{q}$ for all $q=1, \ldots, Q$. A nested $(\boldsymbol{M}, \boldsymbol{N})$-pattern is a phase arrangement over $N$ particle phases in which there are $Q$ distinct symmetric patterns of $M_{q}$ clusters of $N_{q}$ phases. Rather than stabilizing a single $(M, N)$-pattern using the potential $W_{L}^{M, N}$, we stabilize symmetric patterns over connected subgroups of particle phases by combining symmetric phase potentials. Choosing the control

$$
u_{k}=\omega_{0}+\frac{K}{d_{\max }}\left\langle\tilde{L}_{k} \tilde{\boldsymbol{s}}, i e^{i \theta_{k}}\right\rangle-\sum_{q=1}^{Q} \frac{\partial W_{L^{(q)}}^{M_{q}, N_{q}}}{\partial \theta_{k}}
$$

stabilizes a nested $(\boldsymbol{M}, \boldsymbol{N})$-pattern over $B$ separate circular formations of radius $\rho_{0}=\left|\omega_{0}^{-1}\right|$ centered at $c_{0}^{(b)}, b=1, \ldots, B$. Simulations of (1) subject to (40) are shown in Fig. 3(c) for $Q=3$ and $\boldsymbol{M}=\boldsymbol{N}=(2,4,4)^{T}$. In this example, there are three different phase Laplacians given by

$$
\begin{aligned}
& L^{(1)}=\operatorname{diag}\left\{2 P_{2}, 2 P_{2}, 2 P_{2}\right\} \\
& L^{(2)}=\operatorname{diag}\left\{4 P_{4}, 0_{2}\right\} \\
& L^{(3)}=\operatorname{diag}\left\{0_{2}, 4 P_{4}\right\}
\end{aligned}
$$

where $n P_{n}$ is the Laplacian matrix of a complete graph with $n$ nodes and $0_{n}$ is an $n \times n$ matrix of zeros. Each block of two particles is arranged in a $(2,2)$-pattern with phase gains $K_{1}=0.04$ and $K_{2}=-0.01$. The first two particle blocks, i.e., particles with indices $1,2,3,4$, are arranged in a $(4,4)$ pattern. The second and third particle blocks, i.e., particles with indices $3,4,5,6$, are also arranged in a $(4,4)$ pattern. The phase gains for the two $M=4$ patterns are $\left|K_{m}\right|=0.01$ for $m=1, \ldots, 4$. All other parameters are the same as in the previous example.

Note that, in this last example, each pair of particle phases is in the splay state on its own circle, while the four particle phases in the top two circles (and likewise the bottom two circles) are also in the splay state. This example illustrates a pattern that is useful for the mobile sensor network problem. In the ocean sampling application, this pattern is generalized to one in which the three circles are replaced with a stack of three superellipses (approximating rectangles) with coincident inner edges [32]. The pattern provides good sampling coverage of a large rectangular box that bounds the pattern and timely, well distributed sampling in the interior of the box. 


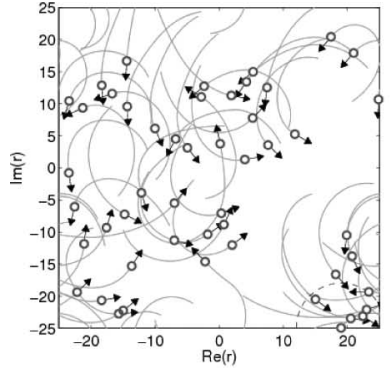

(a)

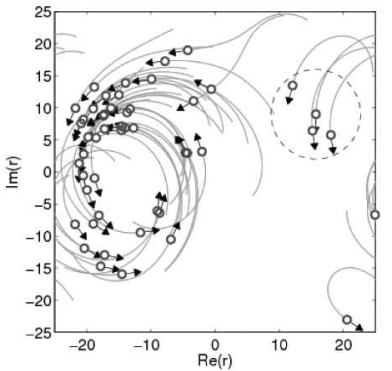

(b)

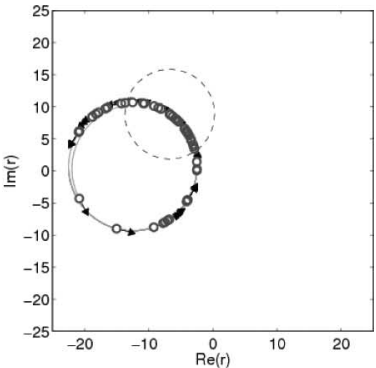

(c)

Fig. 4. Snapshots after 20,75, and 250 time steps of simulation results for $N=50$ particles randomly initialized in a 50 by 50 spatial domain with periodic boundary conditions. The particles are subject to the dynamic control (21) with $K=-0.1$ and a time-varying zonal sensory graph $G(t)$. The radius of proximity $\rho_{k}=7$ for all particles is depicted, for a single particle, as a dashed circle. Setting $\omega_{0}=0.1$ stabilizes circular motion with radius $\rho_{0}=\left|\omega_{0}^{-1}\right|$; setting $\omega_{0}=0$ stabilizes a parallel motion (not shown). (a) $t=20$. (b) $t=75$. (c) $t=250$.

\section{Stability of Circular Collectives With PROXIMITY-BASED COMMUNICATION GRAPH}

In this section, we consider stabilization of collective motion of a network of moving particles with time-varying sensing topologies. We consider sensing to be a form of directed communication in which information flows from the sensed particle to the sensing particle. Accordingly, the edges in a sensory graph are directed from the sensed to the sensing particle. Due to limitations in sensing range, sensing links exist only between neighboring particles, and may change over time as the particles move. We illustrate stabilization of parallel and circular motion with sensory graphs using simulations of the dynamic control law in Theorem 6 for large groups of particles.

Let the sensing topology be described by a time-varying $\delta$ digraph $G(t)=(\mathcal{I}, \mathcal{E}(t), A(t))$. The nonnegative components $a_{k j}(t)$ of the adjacency matrix are bounded away from zero when agent $k$ senses agent $j$ and zero otherwise. We have explored several methods for determining when sensing links are formed. For example, one can construct a sensing graph that models sensors with finite sensing capacity by creating directed links to agent $k$ from a limited number of its nearest neighbors. Let $n_{k}(t)>0$ be the (maximum) number of simultaneously sensed neighbors. In a nearest neighbor sensory graph, agent $j$ is strongly connected to agent $k$ at time $t$, if agent $j$ is one of agent $k$ 's $n_{k}(t)$ nearest neighbors. Note that although nearest neighbor graphs are not strongly connected, the in-degree of each agent is always positive since it equals $n_{k}(t)$.

Another type of sensory graph can be constructed using sensing zones to determine when sensing links are formed. For example, a circular sensing zone centered on agent $k$ is defined by

$$
\mathcal{Z}_{k}(t)=\left\{r \in \mathbb{C}|| r-r_{k}(t) \mid \leq \rho_{k}(t)\right\}
$$

where the radius of proximity $\rho_{k}(t)$ represents the (maximum) sensing range of particle $k$. In a zonal sensory graph, agent $k$ senses agent $j$ at time $t$ if $r_{j}(t) \in \mathcal{Z}_{k}(t)$, where $\mathcal{Z}_{k}(t)$ is agent $k$ 's sensing zone. Note that sensing zone (41), in the case $\rho_{k}=\rho$ for all $k$, gives rise to bidirectional sensing links because of its radial symmetry. Directed sensing links can occur if the sensing zone is not radially symmetric, e.g., if the $\rho_{k}$ are not identical or if the zone contains a "blind spot." The sensing zones, which may be time-varying, need not be identical, and the $k$ th zone need not contain agent $k$.

If $G(t)$ is uniformly connected, the dynamic control (21) enforces convergence of the particle model (1) to a parallel formation if $\omega_{0}=0$ and a circular formation of radius $\rho_{0}=$ $\left|\omega_{0}^{-1}\right|$ if $\omega_{0} \neq 0$. Uniform connectedness of $G(t)$ is not enforced in a sensory graph. In the simulation results given later, we observe that uniformly connected subgroups of particles in a zonal sensory graph exhibit collective motion. The number of subgroups is determined in part by the size of the spatial domain and the radius of proximity.

Fig. 4 shows snapshots after 20,75 , and 250 time steps of simulation results for $N=50$ particles randomly initialized in a 50 by 50 spatial domain with periodic boundary conditions, i.e., horizontal edges of the domain are identified and vertical edges are identified. The particles are subject to the dynamic control (21) with $K=-0.1$ and a time-varying zonal sensory graph $G(t)$. The radius of proximity $\rho_{k}=7$ for all $k=1, \ldots, N$ is depicted, for a single particle, as a dashed circle. Setting $\omega_{0}=$ 0 stabilizes parallel motion (not shown) and setting $\omega_{0}=0.1$ stabilizes circular motion with radius $\rho_{0}=\left|\omega_{0}^{-1}\right|$ (see Fig. 4). For these parameter values, we infer that the entire group of particles is most likely uniformly connected. Using the same parameters in a larger spatial domain results in convergence to several disconnected circular formations (not shown).

\section{CONCLUSION}

This paper proposes a design methodology to stabilize isolated relative equilibria in a model of identical, steered particles moving in the plane at unit speed. In recent work [11], we provided Lyapunov-based stabilizing feedback control laws under the assumption of all-to-all communication among particles. In the present paper, we recover these results under more realistic assumptions on the communication topology.

To address the design of stabilizing feedback in the framework of time-invariant and undirected communication topologies, we generalize the previously proposed potentials in such a way that the derived stabilizing feedback satisfies the communication constraints. The proposed potentials are quadratic forms induced 
by the Laplacian of the communication graph. They reduce to our previously proposed all-to-all potentials in the case of a complete graph.

To address the design of stabilizing feedback in the framework of time-varying and directed communication topologies, we design consensus filters that asymptotically reconstruct the averaged quantities required by all-to-all stabilizing control laws. This approach, recently proposed in [23] for phase models on the $N$-torus, makes it possible to recover the results based on allto-all communication under the sole assumption that the communication graph is uniformly connected. It requires increased communication between communicating particles because particles must exchange relative estimated variables in addition to relative configuration variables.

The proposed results are illustrated in the framework of a sensor network application for ocean sampling [9] that initially motivated most of this research. They are also illustrated in the framework of a proximity-based communication graph, showing how planar collectives can be achieved in a very decentralized way.

The proposed framework provides a fairly complete convergence analysis of collectives achieved in a simple planar particle model. Its extension to more general manifolds has relevant applications such as coordination in space [34]. The recent work [35] should serve as a basis for such extensions. An open question of particular interest is the robustness of the proposed approach to more complicated models for individuals, including dynamic considerations, external disturbances, and discrepancies among the particles.

\section{REFERENCES}

[1] E. Justh and P. Krishnaprasad, "Equilibria and steering laws for planar formations," Syst. Control Lett., vol. 52, no. 1, pp. 25-38, 2004.

[2] J. Fax and R. Murray, "Information flow and cooperative control of vehicle formations," IEEE Trans. Automat. Control, vol. 49, no. 9, pp. 1465-1476, Sep. 2004

[3] W. Ren and R. Beard, "Formation feedback control for multiple spacecraft via virtual structures," IEE Proc.-Control Theory Appl., vol. 151, no. 3, pp. 357-368, 2004.

[4] L. Chaimowicz, V. Kumar, and F. M. Campos, "A paradigm for dynamic coordination of multiple robots," Auton. Robots, vol. 17, no. 1, pp. 7-21, 2004.

[5] Y. Cao, A. Fukunaga, A. Kahng, and F. Meng, "Cooperative mobile robotics: Antecedents and directions," in Proc. IEEE/RSJ Int. Conf. Intell. Robots Syst., 1995, pp. 226-234.

[6] J. Cortès, S. Martìnez, T. Karatas, and F. Bullo, "Coverage control for mobile sensing networks," IEEE Trans. Robot. Autom., vol. 20, no. 2, pp. 243-255, Apr. 2004.

[7] A. L. Bertozzi, M. Kemp, and D. Marthaler, "Determining environmental boundaries: Asynchronous communication and physical scales," in Proc. Coop. Control: 2003 Block Island Workshop Coop. Control, V. Kumar, N. Leonard, and A. S. Morse, Eds. New York: Springer-Verlag, 2005, pp. 25-42.

[8] E. Fiorelli, N. E. Leonard, P. Bhatta, D. Paley, R. Bachmayer, and D. M. Fratantoni, "Multi-AUV control and adaptive sampling in Monterey Bay," IEEE J. Oceanic Eng., vol. 31, no. 4, pp. 935-948, Oct. 2006.

[9] N. Leonard, D. Paley, F. Lekien, R. Sepulchre, D. Fratantoni, and R. Davis, "Collective motion, sensor networks, and ocean sampling," Proc. IEEE, vol. 95, no. 1, pp. 48-74, Jan. 2007.

[10] E. Justh and P. Krishnaprasad, "A simple control law for UAV formation flying,” Inst. System Res., Univ. Maryland, College Park, MD,Tech. Rep. 2002-38, 2002.

[11] R. Sepulchre, D. Paley, and N. Leonard, "Stabilization of planar collective motion: All-to-all communication," IEEE Trans. Automat. Control, vol. 52, no. 5, pp. 811-824, May 2007.
[12] J. Marshall, M. Broucke, and B. Francis, "Formations of vehicles in cyclic pursuit," IEEE Trans. Automat. Control, vol. 49, no. 11, pp. 1963-1974, Nov. 2004.

[13] D. Paley, N. Leonard, and R. Sepulchre, "Collective motion of selfpropelled particles: Stabilizing symmetric formations on closed curves," in Proc. IEEE 45th Conf. Decision Control, San Diego, CA, 2006, pp. 50675072.

[14] J. Jeanne, D. Paley, and N. Leonard, "On the stable phase relationships of ring-coupled planar particles," in Proc. Joint 44th IEEE Conf. Decision Control Eur. Control Conf., Seville, Spain, 2005, pp. 3929-3934.

[15] V. D. Blondel, J. M. Hendricks, A. Olshevsky, and J. N. Tsitsiklis, "Convergence in multiagent coordination, consensus, and flocking," in Proc. Joint 44th IEEE Conf. Decision Control Eur. Control Conf., Seville, Spain, 2005, pp. 2996-3000.

[16] L. Moreau, "Stability of multiagent systems with time-dependent communication links," IEEE Trans. Automat. Control, vol. 50, no. 2, pp. 169-182, Feb. 2005.

[17] A. Jadbabaie, J. Lin, and A. S. Morse, "Coordination of groups of mobile autonomous agents using nearest neighbor rules," IEEE Trans. Automat. Control, vol. 48, no. 6, pp. 988-1001, Jun. 2002.

[18] R. Olfati-Saber and R. Murray, "Consensus problems in networks of agents with switching topology and time-delays," IEEE Trans. Automat. Control, vol. 49, no. 9, pp. 1520-1533, Sep. 2004.

[19] R. Olfati-Saber and J. Shamma, "Consensus filters for sensor networks and distributed sensor fusion," in Proc. Joint 44th IEEE Conf. Decision Control Eur. Control Conf., 2005, pp. 6698-6703.

[20] L. Moreau, "Stability of continuous-time distributed consensus algorithms," in Proc. 43rd IEEE Conf. Decision Control, 2004, pp. 3998-4003. For extended version see: http://arxiv.org math.OC/0409010

[21] A. Jadbabaie, N. Motee, and M. Barahona. "On the stability of the Kuramoto model of coupled nonlinear oscillators," in Proc. Amer. Control Conf., 2004, vol. 5, pp. 4296-4301. For corrected version see: http://www.seas.upenn.edu/jadbabai/papers/ Kuramoto_reallyfinal.pdf

[22] R. Sepulchre, D. Paley, and N. Leonard, "Group coordination and cooperative control of steered particles in the plane," in Group Coordination and Cooperative Control (Lecture Notes in Control and Information Sciences Series 336), K. Y. Pettersen, J. T. Gravdahl, and H. Nijmeijer, Eds. London, U.K.: Spinger-Verlag, 2006, pp. 217-232.

[23] L. Scardovi and R. Sepulchre, "Collective optimization over average quantities," in Proc. 45th IEEE Conf. Decision Control, San Diego, CA, 2006, pp. 3369-3374.

[24] L. Scardovi, A. Sarlette, and R. Sepulchre, "Synchronization and balancing on the $N$-torus," Syst. Control Lett., vol. 56, no. 5, pp. 335-341, 2007.

[25] D. P. Spanos, R. Olfati-Saber, and R. M. Murray, "Dynamic consensus for mobile networks," presented at the presented at the 16th IFAC World Congr., Prague, Czech Republic, 2005.

[26] F. R. K. Chung, Spectral Graph Theory (Regional Conf. Series Mathematics). Providence, RI: American Mathematical Society, 1997, vol. 92.

[27] P. J. Davis, Circulant Matrices. New York: Wiley, 1979.

[28] S. H. Strogatz, "From Kuramoto to Crawford: Exploring the onset of synchronization in populations of coupled oscillators," Physica D, vol. 143, pp. 1-20, 2000.

[29] Y. Kuramoto, Chemical Oscillations, Waves, and Turbulence. New York: Springer-Verlag, 1984.

[30] E. Brown, J. Moehlis, and P. Holmes, "On phase reduction and response dynamics of neural oscillator populations," Neural Comput., vol. 16, no. 4, pp. 673-715, 2004.

[31] R. A. Freeman, P. Yang, and K. M. Lynch, "Stability and convergence properties of dynamic average consensus estimators," in Proc. IEEE Conf. Decision Control, Dec. 2006, pp. 338-343.

[32] D. Paley, F. Zhang, and N. E. Leonard. "Cooperative control for ocean sampling: The Glider coordinated control system," to appear in IEEE Trans. Control Syst. Technol., [Online]. Available: http://www.princeton.edu/ naomi/publications/2006/TCST06.html

[33] E. Justh and P. Krishnaprasad, "Natural frames and interacting particles in three dimensions," presented at the IPAM Workshop: Swarming Nature Design", Los Angeles, CA, Mar. 2006.

[34] S. Nair and N. E. Leonard, "Stabilization of a coordinated network of rotating rigid bodies," in Proc. IEEE Conf. Decision Control, 2004, pp. 46904695.

[35] E. Justh and P. Krishnaprasad, "Natural frames and interacting particles in three dimensions," in Proc. Joint 44th IEEE Conf. Decision Control Eur. Control Conf., Seville, Spain, 2005, pp. 2841-2846. 


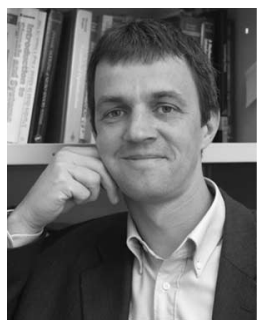

Rodolphe Sepulchre (M'96) received the engineering and Ph.D. degrees in applied mathematics from the University of Louvain, Louvain, Belgium, in 1990 and 1994, respectively.

During 1994-1996, he was a Postdoctoral Fellow at the University of California, Santa Barbara. From 2002 to 2003, he was with Princeton University, Princeton, NJ. He is currently with the Department of Electrical Engineering and Computer Science, Institut Montefiore B28, Université de Liège, Liège, Belgium, as a Professor. His current research interests include nonlinear dynamics and control, collective dynamics and consensus problems on manifolds, and optimization on manifolds. He is the coauthor of the book Constructive Nonlinear Control (Springer-Verlag, 1997). His current research interests include nonlinear dynamical systems.

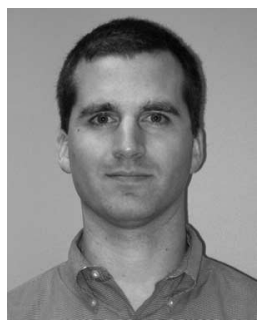

Derek A. Paley (S'02-M'07) received the B.S. degree in applied physics from Yale University, New Haven, CT, in 1997, and the Ph.D. degree in mechanical and aerospace engineering from Princeton University, Princeton, NJ, in 2007.

During 1997-2000, he was an Analyst in the Defense Industry at Metron, Inc., Reston, VA. During 2000-2002, he was a Software Engineer in the Autonomous Underwater Vehicle Industry at Bluefin Robotics Corporation, Cambridge, MA. He is currently an Assistant Professor of Aerospace Engineering at the University of Maryland, College Park. His current research interests include nonlinear dynamics and control, cooperative control of autonomous vehicles, adaptive sampling with mobile sensor networks, autonomous underwater vehicles, and spatial models of biological collectives.

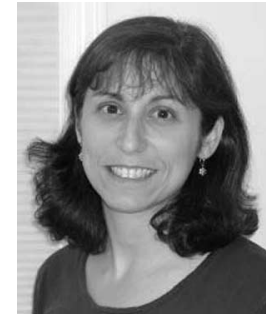

Naomi Ehrich Leonard (F'07) received the B.S.E. degree in mechanical engineering from Princeton University, Princeton, NJ, in 1985, and the M.S. and $\mathrm{Ph} . \mathrm{D}$. degrees in electrical engineering from the University of Maryland, College Park, in 1991 and 1994 respectively.

From 1985 to 1989, she was an Engineer in the Electric Power Industry, MPR Associates, Inc. She is currently a Professor of mechanical and aerospace engineering and an Associated Faculty Member of the Program in Applied and Computational Mathematics, Princeton University. Her current research interests include nonlinear control and dynamics, cooperative control, mobile sensor networks, autonomous underwater vehicles, adaptive ocean sampling, and collective motion in animal aggregations. 